\title{
Retinal photoreceptors of Syrian hamsters undergo oxidative stress during streptozotocin-induced diabetes
}

\author{
A. Colantuoni $^{1}$, B. Longoni ${ }^{1}$, P. L. Marchiafava ${ }^{1}$ \\ ${ }^{1}$ Department of Physiology and Biochemistry, University of Pisa, Pisa, Italy
}

\section{Abstract}

Aims/hypothesis. The aim of this study was to verify whether retinal photoreceptors, like other tissues, are subject to oxidative stress during diabetes.

Methods. Oxidative stress was monitored by the oxidation of preloaded dehydrorhodamine 123 into fluorescent rhodamine 123, during a period of intense illumination of isolated rod retinal receptor cells. These were obtained from 22 Syrian hamsters injected with streptozotocin $(50 \mathrm{mg} / \mathrm{kg}$ body weight., intraperitoneal route) 90 days before the study began. Eleven hamsters were treated daily with melatonin $(0.4 \mathrm{mg} / \mathrm{kg}$ body wt., per os), an antioxidant synthesized within photoreceptors. Isolated photoreceptors were bathed on the stage of a Leitz Orthoplan microscope, where the fluorescent lamp also served as the light stimulus $(485 \mathrm{~nm})$. Fluorescence irradiance was measured by photometry and stored in a personal computer for further analysis.

Results. The light-induced oxidant production greatly decreased and was also delayed in the streptozotocin- injected hamsters compared with the control hamsters matched for age. Similar effects were obtained in control photoreceptors after $40 \mathrm{~min}$ incubation with 2-2'-azobis (2-amidinopropane) dihydrochloride, a potent lipoperoxidation inducer. The effect of melatonin was to partially restore the light-induced fluorescence response.

Conclusion/interpretation. The depression of the light-induced oxidative response in diabetic photoreceptors could be ascribed to a hyperglycaemiainduced background of oxidative stress whereby the light-oxidizable substrate is actually lowered. Melatonin induces a larger fluorescence response during illumination, probably as a consequence of its antioxidant effect during diabetes, which would provide more oxidizable lipids. [Diabetologia (2002) 45: 121124]

Keywords Retinal rod photoreceptor cells, diabetes, lipoperoxidation, oxidative stress, Syrian hamster, melatonin.
Membrane lipid peroxidation at the level of the retina is an early pathological aspect of oxidative stress in diabetes $[1,2]$. Direct evidence of oxidative stress,

Received: 23 September 2001 and in revised form: 27 September 2001

Corresponding author: A. Colantuoni, Department of Physiology and Biochemistry, University of Pisa, Via S. Zeno 31, 56127 Pisa, Italy, e-mail: antcln@dfb.unipi.it

Abbreviations: DHR, Dehydrorhodamine; RHO, rhodamine; STZ, streptozotocin; ABAP, 2-2'-azobis (2-amidinopropane) dihydrochloride; POS, single photoreceptor outer segment; MLT, melatonin however, has not yet been found in diabetic retinal photoreceptors, even though their high physiological production rate of reactive molecular species [3] and their abundant unsaturated fatty acids make these cells a principal substrate for lipid peroxidation.

It has recently been reported that exposure of the outer segments of rod photoreceptor cells, isolated from the frog retina, to bright light of $485 \mathrm{~nm}$ rapidly induces oxidative reactions followed by irreversible cell damage [4]. The light-induced oxidative process depends on a transient state of activated rhodopsin which promotes lipid peroxidation. The oxidative effect of illumination was monitored by the oxidation 
Table 1. Weight, blood glucose, cholesterol, triglycerides, amplitude of fluorescence peak and time to peak of photoreceptor light-induced fluorescence response in age-matched control (CON), age-matched control treated with 2-2'-azobis

\begin{tabular}{|c|c|c|c|}
\hline & $\mathrm{CON}$ & DIA & DIA + MLT \\
\hline $\begin{array}{l}\text { Weight }(\mathrm{g}) \\
n \text { (animals) }\end{array}$ & $\begin{array}{l}160 \pm 7 \\
11\end{array}$ & $\begin{array}{l}128 \pm 10^{*} \\
11\end{array}$ & $\begin{array}{l}132 \pm 9 * \\
11\end{array}$ \\
\hline $\begin{array}{l}\text { Cholesterol (mmol/l) } \\
n \text { (animals) }\end{array}$ & $\begin{array}{l}3.83 \pm 0.46 \\
11\end{array}$ & $\begin{array}{l}8.02 \pm 0.64 * \\
11\end{array}$ & $\begin{array}{l}6.86 \pm 0.31 * \\
11\end{array}$ \\
\hline $\begin{array}{l}\text { Florescence peak (nW) } \\
n \text { (cells) }\end{array}$ & $\begin{array}{l}0.35 \pm 0.04 \\
40 \\
+ \text { ABAP } \\
0.14 \pm 0.05^{*}, * * \\
22\end{array}$ & $\begin{array}{l}0.04 \pm 0.01 * \\
40\end{array}$ & $\begin{array}{l}0.12 \pm 0.03 * * * \\
40\end{array}$ \\
\hline $\begin{array}{l}\text { Time to peak } \\
\text { (s) }\end{array}$ & $120 \pm 15$ & $250 \pm 25^{*}$ & $190 \pm 10 *, * *$ \\
\hline
\end{tabular}

(2-amidinopropane) dihydrochloride $(\mathrm{CON}+\mathrm{ABAP})$, streptozotocin-induced diabetic (DIA) and melatonin-treated diabetic (DIA + MLT) Syrian hamsters

, significantly different compared to control animals (CON)

$* * p<0.01$, significantly different compared to diabetic animals (DIA)

of intracellularly preloaded dehydrorhodamine (DHR) 123 into fluorescent rhodamine (RHO) 123, and by the presence of high levels of thiobarbituric acid reactive substances $[4,5]$.

In this study we used this experimental model to verify directly whether hamster retinal rod photoreceptor cells undergo a light-induced oxidative stress as happens in frogs [4], and whether streptozotocin (STZ)-induced diabetes alters this oxidative process. This investigation is based on the assumption that if, during the course of diabetes, retinal rod photoreceptor cells undergo an increased lipoxygenation, this lipoxygenation might decrease the lipid substrate available for further oxidation by light, thereby reducing light-induced damage to photoreceptor cells and their fluorescence response. According to this hypothesis if melatonin, an antioxidant synthesized in retinal rod photoreceptor cells [4], is given during diabetes, then more oxidizable lipids should be available to enhance the light-induced fluorescence response.

\section{Materials and methods}

A total of 33 male Syrian hamsters, 70-90 g body wt., (Charles River, Calco, Italy) were used: 11 were injected with the vehicle, serving as age-matched controls; 22 were injected with STZ (Sigma Chemical, St. Louis, Mass., USA), $50 \mathrm{mg} / \mathrm{kg}$ body wt., i.p., (DIA group). Eleven were treated with melatonin every day after 15 days (Sigma), $0.4 \mathrm{mg} / \mathrm{kg}$ body wt., per os,
(DIA + MLT group). Blood glucose, cholesterol and triglycerides were measured by colorimetric methods (Roche Diagnostics, Monza, Italy) on blood samples withdrawn from the heart under light thiopental anesthesia (Pentothal Sodium, Gellini, Aprilia, Italy), $30 \mathrm{mg} / \mathrm{kg}$ body wt., i. p., before and 90 days after STZ administration. Hamsters were kept and killed in accordance with principles of laboratory animal care and the institutional rules for care and handling of experimental animals.

Single photoreceptor outer segments (POS) were isolated from dark-adapted retinas 90 days after STZ injection and placed on a Petri dish filled with Locke's solution containing (in mmol/l): $\mathrm{NaCl}, 140 ; \mathrm{KCl}, 3.6 ; \mathrm{CaCl}_{2}, 1.2, \mathrm{MgCl}_{2}, 2.4 ; \mathrm{HE}-$ PES, 10, Glucose, 10, at pH 7.6 [3]. Microscopic analysis did not reveal any morphological difference between retinal rod photoreceptor cells isolated from control and STZ-injected hamsters. DHR 123 (Molecular Probes, Eugene, Ore., USA), which in the presence of reactive oxygen species is oxidized into fluorescent RHO 123 [6], was added to the cell-containing suspension at a final concentration of $10 \mu \mathrm{mol} / \mathrm{l}$, and allowed to diffuse for $20 \mathrm{~min}$ at $37^{\circ} \mathrm{C}$. A drop of the cell suspension was then placed on a microscope slide for observation under fluorescence illumination. Fluorescent microscopy was carried out by epiilluminating POS with the mercury arc lamp (OSRAM) of a Leitz Orthoplan microscope, filtered at $485 \mathrm{~nm}$ $(+/-8 \mathrm{~nm})$ for excitation, and at $520(+/-8 \mathrm{~nm})$ for emission. The same light used for RHO 123 excitation was also used for POS light stimulation (a circle of $20 \mu \mathrm{m}$ diameter, $4.2 \cdot 10^{4} \mathrm{ph}$ $\mathrm{s}^{-1} \mathrm{~cm}^{-2}$ ). A photometric detector (818 ST, Newport, Irvine, Calif., USA), applied to the microscope, measured the irradiance from the POS and fed results into an A/D converter (Labmaster, Axon Instruments, Foster City, Calif., USA) for further computer-assisted data analysis (Origin, MicroCal Software, Northampton, Mass., USA).

Twenty minutes before incubation with DHR 123, 2-2'azobis (2-amidinopropane) dihydrochloride (ABAP) (Wako Chemicals, Dallas, Tex., USA), a water-soluble initiator of lip- 


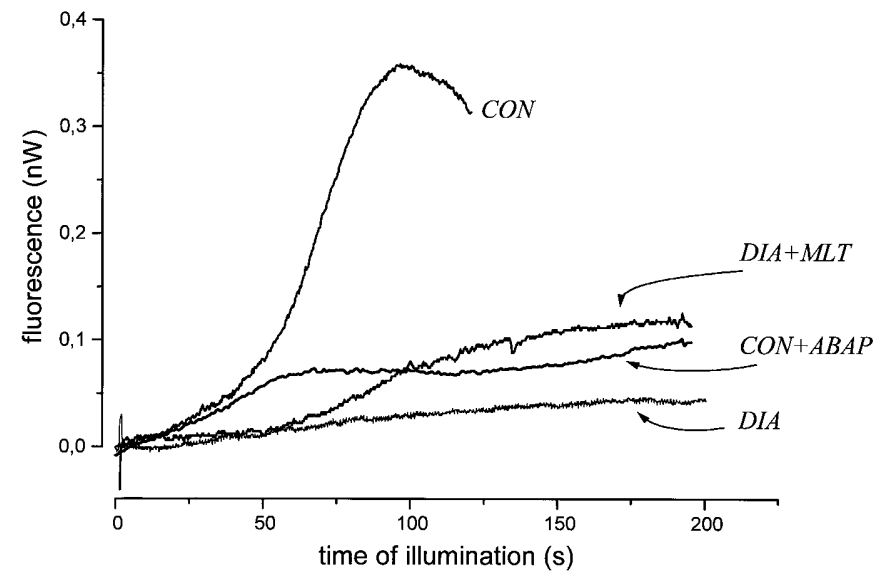

Fig. 1. Typical photometric recordings of light-induced rhodamine 123 fluorescence in isolated outer segment of retinal rod photoreceptor cell in normal (CON) and streptozotocin-induced diabetic (DIA) Syrian hamsters. CON + ABAP: recording from a control cell treated with 2-2'-azobis (2-amidinopropane) dihydrochloride; DIA + MLT: recording from a cell of a streptozotocin-induced diabetic Syrian hamster treated with melatonin. Abscissa indicates the time of illumination. Ordinate represents the output of the recording phototube applied to the microscope

id peroxidation [7], was added to CON POS-containing suspensions ( $\mathrm{CON}+\mathrm{ABAP}$ group, $n=22$ cells) at a final concentration of $2.5 \mathrm{mmol} / \mathrm{l}$.

Data were analysed for statistical significance using the Mann-Whitney and Kruskal-Wallis non-parametric tests. The reported values are means \pm SEM; differences were considered to be statistically significant at $p<0.05$.

\section{Results}

Animal weight, blood glucose, cholesterol and triglycerides all showed a successfully developed experimental diabetes at 90 days after STZ injection (Table 1). The 75-day-long treatment with melatonin did not seem to significantly reduce any of these clinical characteristics.

The control group showed a typical light-induced fluorescence increase recorded photometrically from the POS, reflecting the production of oxidant species during illumination (Fig. 1). The light-induced oxidation slowly rises during an initial phase of $50-70 \mathrm{~s}$, followed by a steeper increase, peaking at about $120 \mathrm{~s}$.

In the diabetic group, diabetes greatly reduced and delayed the light-induced fluorescence response (Fig.1). To test the hypothesis that the reduction observed in the fluorescence response resulted from oxidative stress during diabetes, lipid peroxidation was artificially initiated by adding ABAP $(2.5 \mathrm{mmol} / \mathrm{l} \mathrm{fi-}$ nal concentration), $40 \mathrm{~min}$ before the illumination of POS isolated from the group of control animals. In the control group with ABAP, the antecedent ABAP-induced lipid peroxidation reduced the peak amplitude of the fluorescence response to illumination (Fig. 1).

Melatonin, administered to diabetic hamsters from 15 days after STZ injection, partially restored the fluorescence response to light (Fig. 1).

The fluorescence peak amplitude and the time to peak of the diabetic group, the control group with ABAP and the diabetic group with MLT were significantly different from the control group (Table 1). Furthermore, a significant difference was observed between the diabetic group and the diabetic group with MLT.

\section{Discussion}

Our data indicate oxidative stress in Syrian hamster retinal rod photoreceptor cells during diabetes whereby the light-induced oxidative response almost completely disappears. This result could be due to a change in the phospholipid fatty acid composition because of long-standing lipoxidation in photoreceptors, which is known to occur in other tissues during diabetes [8]. This process could be particularly damaging for retinal rod photoreceptor cells because of their specific membrane phospholipid composition, their high and steady production rate of oxyradicals deriving from a sustained energy demand [3] and the deterioration of the G-protein complex [9] during oxidative stress, thus favouring cytotoxicity.

The experiment with ABAP, where lipid peroxidation was initiated before light stimulation, confirms that a pre-existing oxidative stress could reduce the subsequent light-induced oxidative response.

The suggestion that oxidative stress develops in retinal rod photoreceptor cells during diabetes is further supported by the effects of melatonin, recently shown to be a potent antioxidant synthesized in these cells $[4,10]$. Melatonin, by inhibiting, at least partially, oxidative stress during diabetes, an effect possibly associated with up-regulation of anti-oxidative and/ or anti-apoptotic genes [11], might preserve more polyunsaturated fatty acids which are subsequently oxidized by light. Further studies are needed to show these mechanisms. This protective effect of melatonin argues against the possibility that a reduced amount or function of rhodopsin accounts for the disappearance of the photooxidative response.

However, a variation in the phospholipid fatty acid composition, in addition to altering their susceptibility to oxidation, might also reduce the retinal capacity for photon absorption [12]. Ongoing analytical studies will verify the expected changes in the relative lipid composition of photoreceptors during STZ-induced diabetes.

The alterations in membrane lipid metabolism might be responsible for functional disorders in diabetic retinal rod photoreceptor cells; in this case the 
protective effects of antioxidants might prevent visual diabetic complications.

\section{References}

1. Baynes JW, Thorpe SR (1999) Role of oxidative stress in diabetic complications. A new perspective on an old paradigm. Diabetes 48: 1-9

2. Robison WG Jr, Jacot JL, Katz ML, Glover JP (2000 ) Retinal vascular changes induced by the oxidative stress of alpha-tocopherol deficiency contrasted with diabetic microangiopathy. J Ocul Pharmacol Ther 16: 109-120

3. Demontis GC, Longoni B, Gargini C, Cervetto L (1997) The energetic cost of photoreception in retinal rods of mammals. Arch Ital Biol 135: 95-109

4. Marchiafava PL, Longoni B (1999) Melatonin as an antioxidant in retinal photoreceptors. J Pineal Res 26: 184-189

5. Longoni B, Boschi E, Demontis GC, Marchiafava PL, Mosca F (1999) Regulation of Bcl-2 protein expression during oxidative stress in neuronal and in endothelial cells. Biochem Biophys Res Commun 260: 522-526

6. Royall JA, Ischiropoulos H (1993) Evaluation of 2',7'-dichlorofluorescein and dihydrorhodamine 123 as fluorescent probes for intracellular $\mathrm{H}_{2} \mathrm{O}_{2}$ in cultured endothelial cells. Arch Biochem Biophys 302: 348-355
7. Pryor WA, Cornicelli JA, Devall LJ et al. (1993) A rapid screening test to determine the antioxidant potencies of natural and synthetic antioxidants. J Org Chem 58: 3521-3532

8. Kakkar R, Mantha SV, Radhi J, Prasad K, Kalra J (1998) Increased oxidative stress in rat liver and pancreas during progression of streptozotocin-induced diabetes. Clin Sci (Colch) 94: 623-632

9. Kowluru A, Kowluru RA, Yamazaki A (1992) Functional alterations of $\mathrm{G}$-proteins in diabetic rat retina: a possible explanation for the early visual abnormalities in diabetes mellitus. Diabetologia 35: 624-631

10. Montilla PL, Vargas JF, Tunez IF, Munoz de Agueda MC, Valdelvira ME, Cabrera ES (1998) Oxidative stress in diabetic rats induced by streptozotocin: protective effects of melatonin. J Pineal Res 25: 94-100

11. Osborne NN, Nash MS, Wood JP (1998) Melatonin counteracts ischemia-induced apoptosis in human retinal pigment epithelial cells. Invest Ophthalmol Vis Sci 38: $1390-400$

12. Bush RA, Malnoe A, Reme CE, Williams TP (1994) Dietary deficiency of N-3 fatty acids alters rhodopsin content and function in the rat retina. Invest Ophthalmol Vis Sci 35: $91-100$ 